\title{
Applying Blockchain to Supply Chain Operations at IBM Implementing Agile Practices in a Smart City Environment
}

\author{
Jesús M. Montes ${ }^{1}$, Victor M. Larios ${ }^{2}$, Manuel Avalos ${ }^{1}$, Cecilia E. Ramírez ${ }^{3}$ \\ ${ }^{1}$ International Business Machine Corporation, Guadalajara, \\ Mexico \\ ${ }^{2}$ University of Guadalajara, Guadalajara, Smart Cities Innovation Center CUCEA, \\ Mexico \\ ${ }^{3}$ Division of Industrial Process, Technical University of Jalisco, Guadalajara, \\ Mexico \\ \{jesusmd,jmavalos\}@mx1.ibm.com, victor.m.lariosrosillo@ ieee.org, \\ cecilia.ramirez@utj.edu.mx
}

\begin{abstract}
Nowadays, performance and promptness in the production line are significantly relevant; solutions are being delivered each quarter lead by the evolving innovation of technology which is moving at a fast paste. Taking into consideration concepts from Smarter Cities, Smart Contracts, and Blockchain, we will include these tools in the daily operations of International Business Machines' supply chain to reduce these transaction times radically, implementing agile practices. Fundamentally using IBM Bluemix ${ }^{\circledR}$, which is a fast-growing Platform that provides support for development organizations by defining various users and roles in the cloud environment. IBM Bluemix ${ }^{\circledR}$ provides an extensive inventory of runtime and services that enterprises and developers can use in cloud applications without requiring significant development effort. We as developers can quickly build applications with a choice of several runtimes as can be needed in Supply Chain operations. Furthermore, as the workloads increase, applications can promptly scale ondemand to add more memory or processing power to ensure an optimal block chain experience application. Supply Chain operations can now shift their client-side code into a cloud system environment to leverage service integration, internal confidential activities and services, cloud data and the infrastructure capabilities. It will allow operations to be more productive and highly available and flexible, achieving efficient and sustainable development based on the analysis of real-time information shared by stakeholders and connected devices in a Smart City Environment. IBM is seen as a leader in blockchain, from recent surveys it is revealed that IBM is leading the way of blockchain technology, with more than $40 \%$ of respondents raking it number one. International customers are working with IBM to introduce Blockchain to their businesses. These customers are currently testing out IBM's blockchain technology to help small/medium sized enterprises become more evident and attract investment. Another result of working with IBM is that the customers
\end{abstract}




\begin{abstract}
can track and manage their paper trail from their global shipping process. Another technology that is thriving is artificial intelligence, and in one way or another can influence blockchain, taking into consideration scalability; blockchain grows at a steady pace of megabytes per minutes, here is where AI can introduce decentralized learning systems or new data sharing techniques to make a more efficient system. Another relationship between Blockchain and AI is their effectiveness with security, but AI makes an excellent ally for blockchain to guarantee secure deployments of applications, specifically with the given fixed structure of the systems.
\end{abstract}

Keywords: blockchain, supply chain, smarter cities, smarter contracts, IBM Bluemix ${ }^{\circledR}$, agile practices, artificial intelligence.

\title{
1 Introduction
}

The accomplishment of Smart services by Supply Chain Operations which are the essential elements of a Smart City since they sustain the realization of urban "intelligence" regarding people, industry, economy, governance, environment, mobility, health and living. Smart services aim to improve the quality of life within a city and in this respect to improve "livability" [1]. The types and purposes of Smart Services cannot be predetermined since they are the outcome of innovation, which cannot be decided either without the management of sensitive information that must be kept in log files on an untrusted computer. Service, Supply Chain Operations and Manufacturing areas embraced in investigating manufacturing for design, planning, control, and improvement of services, including distribution operations. Inside the production line, topics include the preparation of production, process design, projects, and personnel, capacity planning, production control, resource planning, quality control, facility layout, materials handling, and new product and process development. Within services, topics include service system design and oversight, service quality, capacity planning, workforce planning, and demand management. Supply chain operations include studies of replenishment policies, inventory and logistics and distribution network design and administration, purchasing, supply management and channel coordination [2, 4]. If an attacker captures Supply Chain Operations data from a machine, it is imperative to guarantee that they will gain little to no information from the log files and to limit their ability to corrupt the log files.

For IBM, Supply Chain Operations are managed to service focusing on the margindevelop procedures and high-cost regions of our clients' value chains, including product development, Supply Chain Planning, Procurement, and Logistics. We deliver valuebased, industry-oriented offerings that contribute to operational improvements and financial gains as depicted in Fig. 1.

Our Clients value chains in a Smart City are frequently merge to an entity named commerce that enables businesses to make Smarter-souring decisions in real time and give their customers the best buying experience possible. We provide Smart Solutions that help organizations deliver engaging customer experiences by intelligently processing and fulfilling products orders most efficiently and profitably possible. For instance, retailers, wholesalers, manufacturers and software developers today are 
challenged by Smart Commerce to meet the demands of empowered consumers; who require advanced delivery methods such as "buy online and pick-up in store." ECommerce is under increasing pressure to offer these flexible options across more channels to meet their customer's expectations and our client's expectations becoming Smart Business. Without Blockchain into the complete Supply Chain OperationsManagement, businesses are forced to make snap decisions based on the sensitive information. Well known in the Informartion Technology field as granular data, resulting in inefficient fulfillment processes, shipping methods, and inventory positions far along as should be in a Smart City Environment being disadvantageous in the broadest commerce industry as seen in Fig. 2.

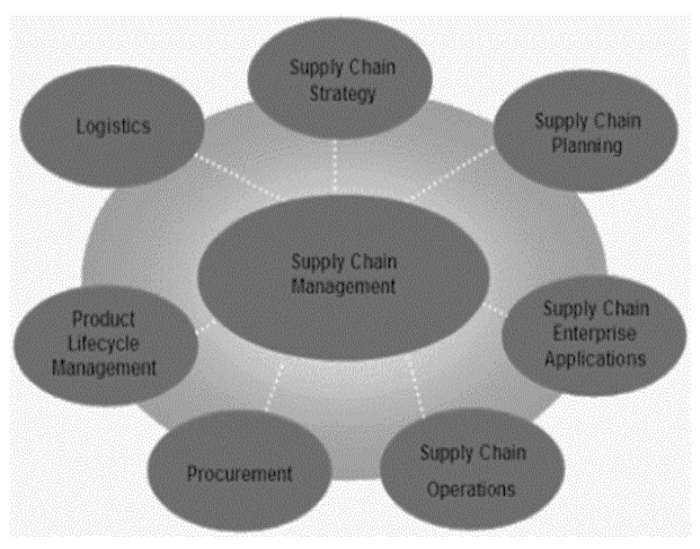

Fig. 1. IBM Supply Chain Management long established [5].

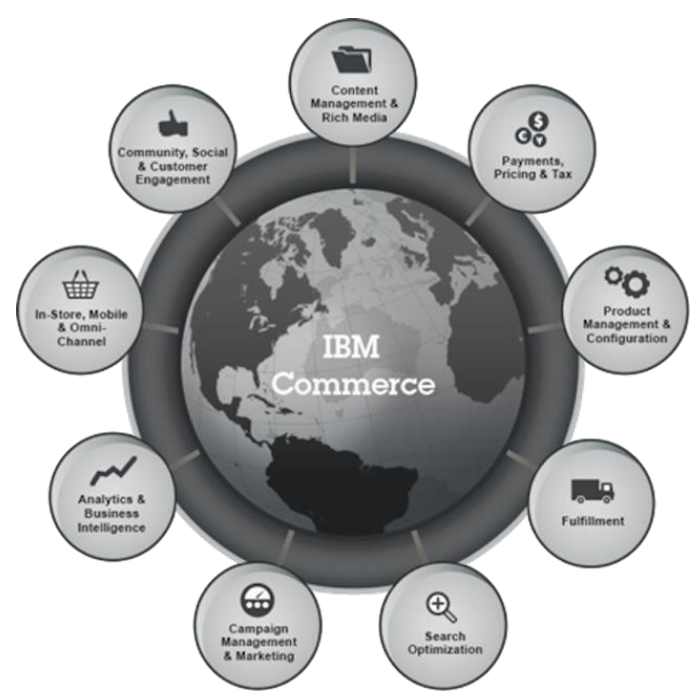

Fig. 2. IBM Commerce Supply Chain Operations in Smart Cities [6]. 
Today, involved across the world are smart cities with the contribution of smart solutions, developed by multidiscipline sciences, which vary from politics and government, to health and education, or to construction and city facilities (water, heat, energy, transportation) [7].

As a result, this compound smart city context has engaged almost all traditional industries that require applying Blockchain in their Supply Chain Operations; blockchain is mostly a database, an extensive network, known as a distributed ledger, which allows any person with access to view and participates in the records ownership and value. The system is updated and verified through consensus of all the parties involved. When adding something it cannot be altered and if it looks valid to everyone, the update is approved [8].

The Blockchain arises as an innovative tool which proves to be useful in some application scenarios. Some large industrial players, such as IBM, Microsoft, Intel, and NEC, are currently investing in exploiting the Blockchain to enrich their portfolio of products. Some researchers and practitioners speculate that the Blockchain technology can change the way we see some current online applications; though it is still early to tell for sure, the Blockchain will fuel substantial changes to a large number of products and will positively impact the digital experience of many individuals around the world [9].

Regarding governance, the growth of smart cities is increasing the use of Information and Communication Technologies (ICTs), that require ameliorating political participation implement public policies or providing public sector services. By doing so, traditional processes of governing do not seem to be appropriated to manage the cities, and therefore new and innovative forms of governance are needed. Because it appears to be clear that transforming public processes will only be achieved with better urban management applying Blockchain to Supply Chain Operations to establish a new governance model for smart cities named as "smart governance." Consequently, governments in smart cities are called to play a vital role in promoting and developing smart cities, using efficient Supply Chain Operations to improve a Smart City Environment [10].

\section{Background}

Throughout the centuries cryptosystems have been used by the military and by the consular services. The nowadays widespread use of computer controlled communication systems in industry or by civil services, often asks for special protection of the data using cryptographic techniques. From the time when storage, and later data recovery, is viewed as a transmission of this data in real-time, we shall always use the term transmission when discussing a situation when information is stored and transmitted [11].

Security systems that protect privacy, secure electronic commerce transactions, cryptography, do not directly prevent fraud. Instead, they detect attempts at fraud after the fact, provide evidence of that fraud to convict the guilty in a court of law, and assume that the legal system will provide a "back channel," currently renowned as blockchain to deter further attempts [12]. 
Applying Blockchain to Supply Chain Operations at IBM Implementing Agile Practices...

Satoshi Nakamoto conceptualized the first blockchain in 2008 and implemented in the following year as a critical element of the digital currency known as Bitcoin, where it serves as the public ledger for all transactions [13].

Over the use of a peer-to-peer network and a distributed timestamping server, a blockchain database can befall management autonomously.

The creation of the blockchain technology for Bitcoin made it the first digital currency to solve the double spending problem. The Bitcoin scheme has been the inspiration for other applications [14].

The blockchain is an essential topic in supply chain management research and practice. For manufacturing companies like IBM, one of the most pressing challenges associated with blockchain in supply chain management is that they typically do not possess sufficient information on what is occurring in the compound supply chain environment, as demonstrated by numerous incidents lacking blockchain. Using eight in-depth case studies across four industries and elaborating on information processing theory, we identify three forms of applying blockchain related uncertainty that each firm faces in the supply chain. Refer to as supply chain uncertainty, source uncertainty, and task uncertainty. The study displays that the extent to which these changes translate into information processing needs depends on a newly identified boundary condition labeled uncertainty intolerance. On the management of such information processing requirements, previous research has pointed primarily at matching information processing requirements with fitting information processing capacity and secondly at mitigating information processing needs with corrective measures. In doing so, this research exemplifies how applying blockchain in the supply chain management may ultimately turn from an adjustment to a firm's daily business to a decisive factor for shaping future supply chains.

Also, the study constitutes a nascent step to elevate information processing theory to the supply chain level. It may contribute to establishing Blockchain in Supply chain operations in Smart Services that a smart city can deliver: Smart Water, Smart Energy, Smart Transportation, Smart Healthcare, Safety/Emergency, Education and Tourism, Smart Waste Management, Smart Buildings, e-government and e-business supported by IBM Bluemix services and implementing agile practices. In the software business, the Agile movement has produced much discussion since its beginning at the 2001 meeting in Snowbird, Utah. Although Agile methods have been applied quite widely in various software development environments, the field of Blockchain in Supply Chain Operations has not been seen in agile projects [15].

\section{Theoretical Framework}

The protection of classified information against unauthorized access or fraudulent changes has been of prime concern throughout the times. Current communication techniques, using computers connected through networks, make all data even more vulnerable to these threats. Since its beginning in 2009, Bitcoin's blockchain has fueled innovation and some new applications, such as smart contracts, have been intended to take advantage of the blockchain [16]. Further concerns have come up that were not relevant beforehand, e.g., how to append a digital signature to a digital document in such a manner that the signer cannot deny later that the paper was signed by him/her [17]. 
Cryptology addresses the above issues. It is at the base of all information security. The techniques engaged to this end have become increasingly mathematical of nature. The study of cryptosystems, cryptology, can be subdivided into two regimens. Cryptography deals with the design of cryptosystems, while cryptanalysis considers the breaching of cryptosystems. These two aspects are closely related; when setting up a cryptosystem the analysis of its security plays an important role [18].

A blockchain database contains two kinds of records: transactions and blocks. Blocks held batches of hashed logical operations and encoded into a Merkle tree as depicted in Fig. 3. Each block comprehends the hash of the previous block in the blockchain, linking the two. Variants of this format were used previously, for example in Git, and it is not by itself sufficient to qualify as a blockchain. The linked blocks form a chain; constant process ratifies the integrity of the previous block, all the way back to the source block. Division structures block into layers defined by protocols [19].

Some blockchains create a new block as rapidly as every five seconds. As blockchains phase, they are sometimes separate blocks that can be validated concurrently, creating a provisional fork. Now with a secure hash based history, all blockchains have a specified algorithm for storing different versions of the history so that one selected with a higher value over others. Unselect blocks for inclusion in the chain are called orphan blocks [20].

Peers supporting the database do not have the same version of the history. Instead, they keep the highest scoring version of the database that they currently know.

Each time a peer receives a higher scoring version, then generally the previous version with a single new block added, is extended or it overwrites their database and retransmits the enhancement to their peers. There is never an absolute assurance that any particular entry will remain in the best version of the history always.

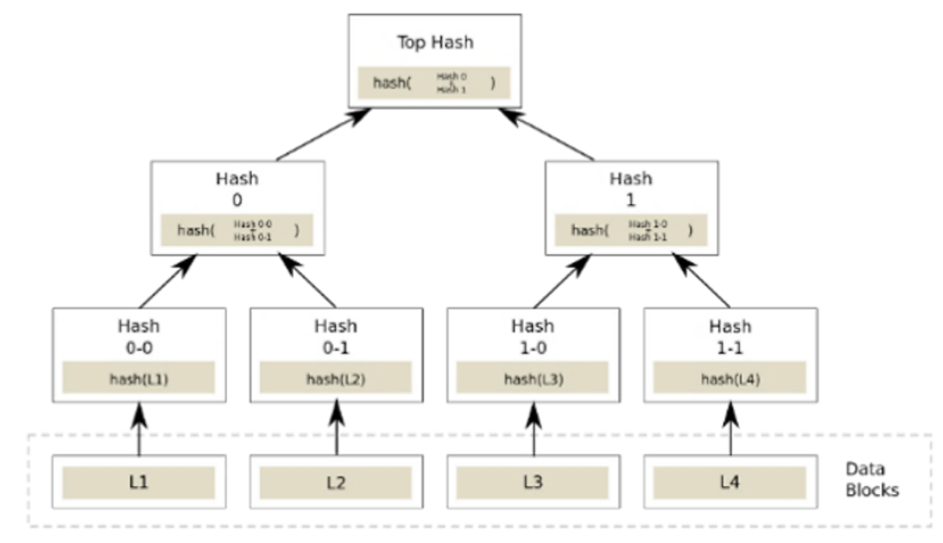

Fig. 3. An example of a Merkle tree [21].

But cause blockchains are characteristically built to sum the score of new blocks onto old blocks, and there are motivations to only work on extending on new blocks instead of overwriting old blocks. The possibility of an entry becoming superseded goes down exponentially, built on top of it are more blocks, eventually becoming very small. 
For instance, in a blockchain using the proof-of-work system, the chain with the most common proof-of-work is considered the valid one by the network all the time.

In practice, some methods can demonstrate a sufficient level of computation. Within a blockchain, the calculation is carried out redundantly rather than in the traditional segregated and parallel manner [22].

\section{$4 \quad$ Methodology}

For this endeavor, the tasks will be broken down into five process groups; initiating, planning, executing, monitoring and controlling, and closing. In which we will apply knowledge, tools, skills, and techniques to launch activities to meet the project's monitoring \& controlling and closing phases [23]. Diving the activities within these groups will give order to our project's research and will allow us to work on any algorithm needed one stage at a time. For this project to be successful, we come across the primary challenge of achieving all of the project goals within the given constraints.

Typically the critical restrictions are scope, time, quality and budget within the projects. These will be our primary focus in the initiating and planning phases, as long as we attend to these requirements in order and fashion, we should have a bright and smooth start in our journey to implement this project. The secondary, and probably more ambitious task is to optimize the assignments of all the essential contributions and apply them to meet predefined objectives, which are set as milestones to acquire a straightforward implementing of agile practices. The practices of continuous integration and test-first programming appear to be practices that can make measurable improvements in the quality and development time for software projects, focusing in [15]:

- Value individuals and interactions over processes and tools.

- Value is working software over comprehensive documentation.

- Value customer collaboration over contract negotiation.

- Value is answering to change over following a plan.

Once we have our path set out, using the project management discipline, we will then need to integrate the Blockchain technology in our supply chain system, for this endeavor, we will work with the IBM Bluemix ${ }^{\circledR}$ platform. Defining IBM Bluemix ${ }^{\circledR}$ as a cloud platform as a service (PaaS). It supports several programming languages and services along with integrated Development Operations to build, run, deploy and manage applications on the cloud. Bluemix ${ }^{\circledR}$ is founded on Cloud Foundry open technology and runs on SoftLayer infrastructure; with Bluemix ${ }^{\circledR}$ we are working with our ledger on the cloud platform, looking to offer solutions for this case study and drive business value with services, applications, and infrastructure. With the cloud resources at our disposition, it will be possible to bring together the data sources for this environment, scale systems, and incorporate customized services to drive the business results quickly and within the budget. 


\section{$5 \quad$ Experimental Results}

Smart Cities require efficient management of a vast number of monitored data regarding processing, storing and analysis is an important matter to deal with for its large-scale adoption in Supply Chain Operations. In a Smart City Environment, any industry is entering a new era of safe and fast transactions with Blockchain technology.

Though they require a robust and scalable high-performance computing and massive storage organization for real-time processing and storing of the data as well as analysis (on-line and off-line), of the processed data under context using integrally complex models to extract information from specific supply chain operations. The adoption of blockchain can lead to performing more enterprise operations, letting the Supply Chain be the turning point. We can see blockchain applied to all departments, and being offered as a solution to the world.

A goal is to document and track all the activities of an average business day in the supply chain, and build our case study; and eventually, apply the improvements produced by the Blockchain technology. The results will be presented in different phases as depicted in Fig. 4.

Adapting planning to business needs, supply chain operations and market situation expectations change so rapidly that the preparation must be verified and modified on an ongoing basis as is shown in Fig. 4, where development needs to be very reactive where short-term goals change often.

Evolutionary development is something nobody can afford to spend months on, to deliver complete applications. Quick time to supply chain is critical even with limited functionality. Following the initial delivery, the Bluemix application goes through an ongoing refinement to meet all required features and to satisfy continuous flow of the supply chain operations requirements.

In the case of an early delivery, we need to advertise and deliver the new service very quickly before competition enters the game. There will be a delivery as soon as there is a Beta quality version. Even though it will not have the full set of functionalities, it will let customers experience some of the benefits of the application and give a feel for what is coming.

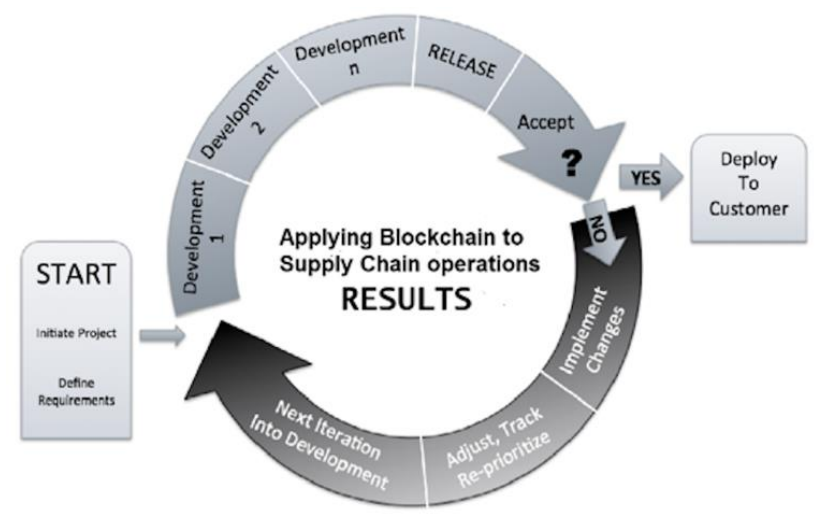

Fig. 4. Applying Blockchain to the supply chain operations [24]. 
Continuous improvement, starting with the Beta Bluemix application, we need to improve the use on an ongoing basis. Bi-weekly or monthly updates to the application will be the goal. Implementation of the updates requires working in non-downtime fashion for business continuity. It will allow for quick defect fixes and provides an ongoing commitment to the supply chain operations.

\section{A. Impact}

To quicken the times of distribution and take advantage of the internet, to have faster online services. These transactions would consist of orders and contracts, making them available to all interested parties instantly and with a legal validity.

Also, this project will aim to impact the process cost, by cutting time in order processing and reducing inventory space with better tracking and logistics that smart cities prioritize establishing local economic growth and taking care of communities with special needs (unemployment, youth, older adults, accessibility and young families). Environmental protection and climate change follow, even if we consider that smart water, smart energy, and smart buildings deal with the same issue. Cities smart ecological performance needs to increase to achieve an urban sustainability vision.

\section{Conclusions and Future Work}

IBM's teams will be interacting with one another, collaborating within the supply chain, Bluemix ${ }^{\circledR}$, and Blockchain, ironing out details, obtaining agreements, asking questions, getting to know each other, and developing trust and bonds. To have face to face interaction, nonetheless, taking into consideration that the most challenging task is still when Supply Chain operations have a high dependence with overseas partners, adding distiance and time barriers to the routin operations. In this new agile world, it is essential to reduce transactions times, be more confidential than we used to have been in the past. From experience learned from how to improve Supply Chain processes applying Blockchain, we can too review and enhance Smart Cities services and procedures as the primary contribution to the field.

This project is ambitious and complex to tackle, there is a lot of theory out there, and very few companies that have successfully applied blockchain to their activities. The project will be a significant challenge and will require identifying entire departments within the Supply Chain process to start with the investigation and application of a case study with project management discipline.

To migrate the supply chain transactions onto Bluemix, our cloud platform, education, and skills will be an obligated requirement. Bluemix ${ }^{\circledR}$ is a fast-growing Platform as a Service (PaaS), in the cloud environment. We will need to get onboard to one of the most critical software development paradigm shifts in the subsequent eras. Online courses offered at IBM, and in-person classes of the subject matter are available nationally and internationally. By the end of the project's research, we have to demonstrate and understand why Bluemix is all we need to prototype, develop and deploy our applications to the supply chain production environment. 
Which can be a natural focus on smart service standardization that will require competitive standards and suggest technical specifications and guidelines for similar solutions development.

Among the elements that these rules try to identify concern smart city services, which conveys the products/services and components that can generate a smart city ecosystem, such as smart transportation, smart water, smart energy, which are the most common prioritize smart city areas.

Acknowledgements. To the IBM experts for their advice. To the University of Guadalajara's academic researchers for tutoring, guidance, and their collaboration. To the Technical University of Jalisco for its knowledge support and availability.

\section{References}

1. Ben, A. et al.: Industrial and Business Systems for Smart Cities. Proceedings of the 1st International Workshop on Emerging Multimedia Applications and Services for Smart Cities (2014)

2. Duenyas, I., Jing-Sheng, S.: Manufacturing, Service, and Supply Chain Operations. Operations Research (2009)

3. Busse, C., Meinlschmidt, J., Foerstl, K.: Managing information processing needs in Global Supply Chains: A prerequisite to sustainable Supply Chain Management. Journal of Supply Chain Management (2017)

4. Adiseshu, H., Lakshman, T.V.: On the Security and Performance of Proof of Work Blockchains. Proceedings of the 15th ACM Workshop on Hot Topics in Networks (2016)

5. https :// www-07.ibm.com/services/hk/bcs/supply_chain_ops.html (2016)

6. http:// www.decidesoftware.com/integrated-Omni-channel-supply-chain-ibm-jda/ (2014)

7. Ghassan, K: On the Security and Scalability of Bitcoin's Blockchain. Conference on Computer and Communications Security (2016)

8. Adiseshu, H., Lakshman, T.V.: The Internet Blockchain: A Distributed, Tamper-Resistant Transaction Framework for the Internet November. Proceedings of the 15th ACM Workshop on Hot Topics in Networks (2016)

9. Maxwell, D., et al.: Effing' the ineffable: opening up understandings of the blockchain. Proceedings of British HCI Conference (2015)

10. Sakamoto, M., Nakajima, T.: Gamifying smart city services to flourish our society. International Joint Conference on Pervasive and Ubiquitous Computing and Proceedings of the ACM International Symposium on Wearable Computers (2015)

11. Miller, A., et al.: The Honey Badger of BFT Protocols. Conference on Computer and Communications Security (2016)

12. Decker, C., et al.: Bitcoin Meets Strong Consistency. Proceedings of the 17 th International Conference on Distributed Computing and Networking (2015)

13. Gervais, A., et al.: On the Security and Performance of Proof of Work Blockchains. Proceedings of the ACM SIGSAC, Conference on Computer and Communications Security (2016)

14. Bogner, A., et al:: A Decentralised Sharing App running a Smart Contract on the Ethereum Blockchain. Proceedings of the 6th International Conference on the Internet of Things (2016)

15. Matthews, C.E.: Agile practices in embedded systems. SPLASH '11, Workshops: Proceedings of the compilation of the co-located workshops on DSM'11 (2011)

16. Judmayer, A., Weippl, E.: Condensed Cryptographic Currencies Crash Course (C5), Proceedings of the ACM SIGSAC Conference on Computer and Communications Security (2016)

17. Henk, C.A., van, T.: Fundamentals of Cryptology: A Professional Reference and Interactive Tutorial. Kluwer Academic Publishers (2000)

18. Underwood, S.: Blockchain beyond bitcoin. Communications of the ACM: 59(11) (2016)

19. Faísca, J.G., Rogado, J.Q.: Decentralized Semantic Identity. Proceedings of the 12th International Conference on Semantic Systems (2016)

20. http:// www .marcoyuen.com/articles/2014/02/01/merkle-tree.html (2014) 
Applying Blockchain to Supply Chain Operations at IBM Implementing Agile Practices...

21. Bogner, A., et al.: A Decentralised Sharing App running a Smart Contract on the Ethereum Blockchain. Proceedings of the 6th International Conference on the Internet of Things (2016)

22. Norta, A.: Conflict-Resolution Lifecycles for Governed Decentralized Autonomous Organization Collaboration. Proceedings of the 2nd International Conference on Electronic Governance and Open Society: Challenges in Eurasia (2015)

23. Project Management Institute: A Guide to the Project Management Body of Knowledge (PMBOK Guide). Global Standard (2013)

24. http://www .code4nord.com/agile-methodology/ (2014) 